Chinese Journal of Organic Chemistry

\title{
乙酸铵催化的酚与多聚甲醛的甲酰化反应研究
}

李 倩 杨 丽刘 伟 王天旳手朱月杰杜正银*
(西北范大学化学化工学院兰州 730070)

\begin{abstract}
摘要 在乙酸铵作为催化剂的条件下，芸酚和多聚甲醛在醋酸溶液中反应，成功合成了一系列羟基菱甲醛，收率最高 达 $86 \%$. 将该方法应用于苯酚及取代苯酚的甲酰化反应，以 $42 \%$ ～ $58 \%$ 的中等收率得到水杨醛类化合物. 根据反应结 果提出了可能的作用机理. 该方法不使用任何金属试剂，具有反应条件温和、操作简单及成本低廉的特点.
\end{abstract}

关键词 荎酚; 苯酚; 多聚甲醛; 甲酰化反应; 绿色合成

\section{Formylation of Phenols and Paraformaldehyde Catalyzed by Ammonium Acetate}

\author{
Li, Qian Yang, Li Liu, Wei Wang, Tianyun Zhu, Yuejie Du, Zhengyin* \\ (College of Chemistry and Chemical Engineering, Northwest Normal University, Lanzhou 730070)
}

\begin{abstract}
The reaction of naphthol and paraformaldehyde in acetic acid solution with ammonium acetate as a catalyst resulted in the synthesis of a series of hydroxynaphthalenes in yields of up to $86 \%$. Several phenols were also transformed into the corresponding salicylaldehydes under the same reaction conditions in moderate yields of $42 \% \sim 58 \%$. A rational mechanism was also proposed based on the facts of experimental observations. This metal-free process has the advantages of mild reaction conditions, simple operation and low cost.
\end{abstract}

Keywords naphthol; phenol; paraformaldehyde; formylation reaction; green synthesis

羟基取代萗甲醛类化合物中含有高反应活性的酚 着基和甲酰基，是农药、医药 ${ }^{[1-3]}$ 、化妆品 ${ }^{[4]}$ 、天然产 物 ${ }^{[5]}$ 及化学传感器 ${ }^{[-9}$ 的重要合成中间体, 被广泛用于纺 织工业 ${ }^{[10]}$ 和染料工业 ${ }^{[11]}$, 在精细有机化工领域具有重 要的地位. 羟基取代䒺甲醛类化合物中具有代表性的是 2-羟基荎-1-甲醛，其常见的合成方法是 Duff 法 ${ }^{[12] 、}$ Reimer-Tiemann 法 ${ }^{[13]}$ 和甲醛法 ${ }^{[14]}$ (Scheme 1). Duff 法是 2-荎酚与六次甲基四胺在强酸性条件下反应, ReimerTiemann 法是菜酚与三氯甲烷在强碱性条件下反应, 反 应条件苛刻, 废弃物较多而且产物收率低. 新的研究大 多都是在这两种方法的基础上进行改良. 1987 年, 马克 荣等 ${ }^{[15]}$ 以 2-芸酚、三氯甲烷和氢氧化钠为底物, 在相转 移催化剂条件下, 采用 Reimer-Tiemann 反应使 2 -羟基萗 -1-甲醛的产率提高到 $51.4 \%$, 相比于无催化剂的 Reimer-Tiemann 反应产率提高了 $13.2 \% .2004$ 年, 夏士 朋等 ${ }^{[16]}$ 采用过量三氯甲烷和氢氧化钠, 并以苠基三甲
基溴化铵作为相转移催化剂合成了 2-羟基萗-1-甲醛, 产率提高至 77\%. 2006 年, 赵胜芳[17]等采取 Duff 法将 2菜酚与六亚甲基四胺在浓硫酸催化下微波加热 $3 \mathrm{~h}$, 制 备了 2-羟基萗-1-甲醛. 这种改进的微波辐射 Duff 法极 大地缩短了反应所需的时间. 2017 年, Yang 等 ${ }^{[18]}$ 以硫酸 作为催化剂, 采用 Duff 法将 2-菜酚和六亚甲基四胺的 乙酸溶液加热搅拌反应 $10 \mathrm{~h}$, 也以 $76 \%$ 的收率得到产物 2-羟基芸-1-甲醛. 上述方法只能用于 2-荎酚的反应, 对 于 1 -蒜酚的甲酰化反应则鲜有报道.

甲醛法是以甲醛水溶液、多聚甲醛为甲酰化试剂的 菜酚甲酰化反应新方法。1999 年, Hofsløkken 和 Skattebø ${ }^{[14]}$ 首先报道了以氯化镁-三乙胺为碱, 2-蒜酚与 多聚甲醛在乙腈中回流反应 $17 \mathrm{~h}$ 后 2-羟基荎-1-甲醛的 收率为 73\%. 2013 年, Shameli 等 ${ }^{[19]}$ 报道在过量甲氧基美 促进下 1-䒬酚和 2-萗酚分别与多聚甲醛进行固相研磨 反应后用硫酸处理能生成相应的邻羟基菜甲醛，收率分

\footnotetext{
* Corresponding author. E-mail: clinton_du@126.com

Received November 9, 2020; revised December 26, 2020; published online January 14, 2021.

Project supported by the National Natural Science Foundation of China (Nos. 21262028, 21762039) and the Natural Science Foundation of Gansu Province (No. 20JR5RA521).

国家自然科学基金(Nos. 21262028, 21762039)和甘肃省自然科学基金(No. 20JR5RA521)资助项目.
} 
别为 70\%和 $60 \% .2018$ 年, Naeimi 和 Zakerzadeh ${ }^{[20]}$ 研究 了在微波辐射条件下, 氧化镁纳米晶催化 2-萘酚与多聚 甲醛的无溶剂甲酰化反应得到 2-羟基䒺-1-甲醛, 但收 率偏低, 仅为 $65 \%$.

(a) Duff method<smiles>[CH+][CH+][CH+]c1ccc2ccc(O)c(C=O)c2c1</smiles>

(b) Reimer-Tiemann method<smiles>O=Cc1c(O)ccc2ccc(O)cc12</smiles>

(c) Formaldehyde method<smiles>O=Cc1ccc2c(C=O)c(O)ccc2c1</smiles>

图式 1 合成 2-羟基-1-萗甲醛的方法

Scheme 1 Synthesis of 2-hydroxy-1-naphthaldehyde

上述 Duff 法、Reimer-Tiemann 法和甲醛法虽然能 以菜酚为原料成功制备出相应的甲酰化产物, 但大多反 应时间长, 产物复杂、收率低, 有的需要金属有机化合 物或强酸强碱等特殊试剂, 设备腐蚀严重, 环境污染也 不容小觑. 因此需要开发环境友好的、高效的荎酚甲酰 化方法.

本课题组长期致力于绿色高效有机合成新方法的 研究 ${ }^{[21]}$, 在前期研究的基础上, 发现在乙酸铵促进下, 萗酚和多聚甲醛发生甲酰化反应, 一步快速、高产率地 合成邻羟基䒺甲醛. 该方法用于苯酚类化合物，也能以 中等收率得到相应产物.

\section{1 结果与讨论}

\section{1 反应条件优化}

为探究促进反应进行的最佳实验条件, 选取 2-䒺酚 和多聚甲醛(或甲醛水溶液)的反应作为模板反应, 着重 从溶剂种类、催化剂种类、催化剂用量和反应时间等条 件进行优化, 实验结果见表 1 . 从表 1 结果可以看出, 以 甲醛水溶液作为甲酰化试剂, 不加入任何催化剂, 直接 在甲醛水溶液中, 或者加入异丙醇、乙醇作为共溶剂时, $50{ }^{\circ} \mathrm{C}$ 反应 $12 \mathrm{~h}$ 几乎得不到任何产物(Entries 1 3); 但 是若以冰乙酸为共溶剂时在同样条件下反应, 目标产物 2-羟基䒺-1-甲醛(2a)的产率为 26\% (Entry 4). 接下来用 多聚甲醛代替甲醛水溶液作为甲酰化试剂, 同样不加催 化剂, 且以冰乙酸作为溶剂时, 在 $100{ }^{\circ} \mathrm{C}$ 反应 $12 \mathrm{~h}$, 产 物的收率提高为 35\% (Entry 5). 因此, 后面的实验选取 多聚甲醛作为酰化剂, 冰乙酸作为反应溶剂, 考察了不 同催化剂对反应的促进作用. 当分别选用 $10 \mathrm{~mol} \%$ 的三 氯化铁和硝酸铈铵作为 Lewis 酸催化剂时. 几乎无产物
生成(Entries 6, 7); 当以氯化铵、四丁基溴化铵(TBAB)、 十六烷基三甲基溴化铵(CTAB)为催化剂时，产物收率 分别为 $38 \% 、 26 \%$ 和 $31 \%$, 催化效果不明显(Entries 8 $10)$; 令人惊喜的是当以乙酸铵作为催化剂, 在 $100{ }^{\circ} \mathrm{C}$ 下反应 $5 \mathrm{~h}$, 产物收率就达到了 70\% (Entry 11). 这可能 是反应液中醋酸铵与冰乙酸能形成缓冲溶液, 使反应体 系有一个稳定的弱酸性 $\mathrm{pH}$ 值范围, 有利于反应的进行. 接下来, 在该条件下考察了催化剂用量对产物收率的影 响. 乙酸铵的用量从 $10 \mathrm{~mol} \%$ 提高到 $50 \mathrm{~mol} \%$ 时, 产率 由 $70 \%$ 升高到 $85 \%$, 但当使用 $100 \mathrm{~mol} \%$ 乙酸铵时, 产率 不再增加(Entries 12 14). 当反应温度从 $100{ }^{\circ} \mathrm{C}$ 降到 $70{ }^{\circ} \mathrm{C}$ 时, 2a 的产率也降低到了 70\% (Entry 15). 因此选 择 $50 \mathrm{~mol} \%$ 的乙酸铵作为该反应的催化剂. 至此得出 Entry 13 中的条件为最佳反应条件. 反应过程中，尽管 多聚甲醛是过量的，荎酚也不会发生多甲酰化反应，而 且羟基邻位发生甲酰化反应的区域选择性为 $100 \%$.

表 1 萗酚与多聚甲醛反应的条件优化 ${ }^{a}$

Table 1 Optimization of reaction conditions

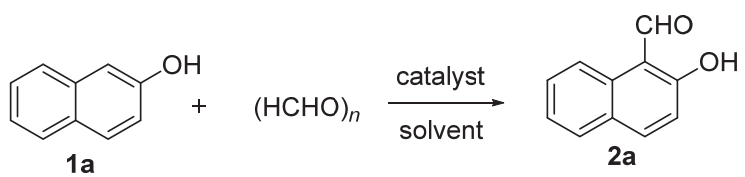

\begin{tabular}{ccccc}
\hline Entry & Catalyst $(\mathrm{mol} \%)$ & Solvent & Time/h & Yield ${ }^{b} \%$ \\
\hline 1 & - & None & 12 & - \\
2 & - & Isopropanol & 12 & Trace \\
3 & - & Ethanol & 12 & Trace \\
4 & - & HOAc & 12 & 26 \\
5 & - & HOAc & 12 & 35 \\
6 & $\mathrm{FeCl}_{3}(10)$ & HOAc & 12 & Trace \\
7 & $\mathrm{Ce}\left(\mathrm{NH}_{4}\right)_{2}\left(\mathrm{NO}_{3}\right)_{6}(10)$ & HOAc & 12 & Trace \\
8 & $\mathrm{NH}_{4} \mathrm{Cl}(10)$ & HOAc & 12 & 38 \\
9 & $\mathrm{TBAB}(10)_{10}$ & HOAc & 12 & 26 \\
11 & $\mathrm{CTAB}(10)^{\mathrm{AcONH}_{4}(10)}$ & HOAc & 12 & 31 \\
12 & $\mathrm{AcONH}_{4}(20)$ & HOAc & 5 & 70 \\
$\mathbf{1 3}$ & $\mathbf{A c O N H}_{4}(\mathbf{5 0})$ & HOAc & 5 & 76 \\
14 & $\mathrm{AcONH}_{4}(100)$ & HOAc & $\mathbf{5}$ & $\mathbf{8 5}$ \\
$15^{c}$ & $\mathrm{AcONH}_{4}(100)$ & HOAc & 5 & 85 \\
\hline
\end{tabular}

${ }^{a}$ Reaction conditions: 2-naphthnol (1a, $\left.0.25 \mathrm{mmol}\right)$, formaldehyde $(1.25 \mathrm{mmol}$ $37 \%$ aqueous solution in Entries 1 4 and paraformaldehyde in Entries 5 14), catalyst $(5 \sim 100 \mathrm{~mol} \%)$ in solvent $(2.5 \mathrm{~mL})$ at $50{ }^{\circ} \mathrm{C}$ (for formaldehyde aqueous solution) or $100{ }^{\circ} \mathrm{C}$ (for paraformaldehyde) for indicated hours until the yield of $2 \mathbf{a}$ does not change. TBAB is $\mathrm{Bu}_{4} \mathrm{NBr}$ and CTAB is $\left(n-\mathrm{C}_{16} \mathrm{H}_{33}\right)-$ $\mathrm{Me}_{3} \mathrm{NBr} .{ }^{b}$ Isolated yield. ${ }^{c}$ The reaction was conducted at $70{ }^{\circ} \mathrm{C}$.

\section{2 反应底物适用性拓展}

为了确定该方法的底物适用范围, 在上述优化条件 下，以菜酚及各种取代的菜酚作为底物，多聚甲醛为酰 化试剂, 合成出了一系列邻羟基萗甲醛类化合物, 结果 见表 2 所示. 首先把 2-䒺酚的优化条件应用于 1-萘酚, 
发现 1-萗酚与多聚甲醛反应也能以 $86 \%$ 的产率生成 1 羟基萗-2-甲醛 $(2 \mathbf{b})$. 当䒺酚的芳环上有 $\mathrm{Br} 、 \mathrm{Cl} 、 \mathrm{NO}_{2}$ 和 $\mathrm{CH}_{3} \mathrm{O}$ 基等取代基时, 取代的 1-萗酚和 2-䒺酚都能以 $100 \%$ 的区域选择性发生邻甲酰化反应生成相应的邻羟 基萗甲醛类产物, 收率在 $62 \% \sim 78 \%(2 \mathrm{c} \sim 2 \mathrm{~g})$. 值得一 提的是, 如果 2-䒬酚的 1-位没有取代基, 那么邻甲酰化 反应发生在 1-位; 如果 2-菜酚的 1-位有取代基, 譬如溴 原子, 那么发生邻甲酰化反应后会生成相应的 4-溴-3羟基䒺-2-甲醛 $(2 \mathrm{e}, 62 \%$ ), 但仍然是羟基的邻位. 令人意 外的是 5-氨基菜酚在上述标准条件下却无任何产物生 成 $(\mathbf{2 h}, 0 \%)$; 以 2,7 -萗二酚为反应底物, 既不生成 1-位单 甲酰化产物, 也没生成 1,8-双甲酰化产物(2i，0\%), 其中 原因尚不清楚，正在进一步研究中.

表 2 䒺酚的适用范围 $a, b$

Table 2 Scope of naphthols

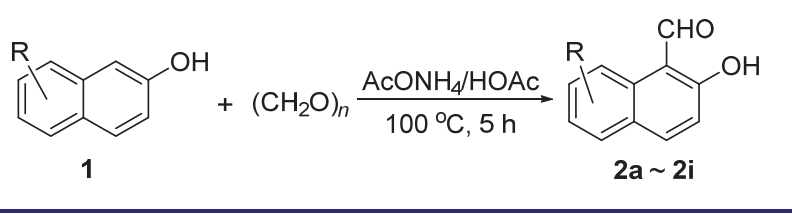<smiles>O=Cc1c(O)ccc2ccccc12</smiles>

2a, $85 \%$<smiles>O=Cc1c(O)cc(Cl)c2ccccc12</smiles>

2d, $76 \%$<smiles>COc1cc(C=O)c(O)c2ccccc12</smiles>

2g, $74 \%$<smiles>O=Cc1ccc2ccccc2c1O</smiles>

2b, $86 \%$<smiles>O=Cc1cc2ccccc2c(O)c1O</smiles>

$2 e, 62 \%$<smiles>Nc1cccc2c(O)c(C=O)ccc12</smiles>

2h, $0 \%$<smiles>O=Cc1c(O)ccc2cc(Br)ccc12</smiles>

2c, $78 \%$<smiles>O=Cc1cc([N+](=O)[O-])c2ccccc2c1O</smiles>

2f, $78 \%$

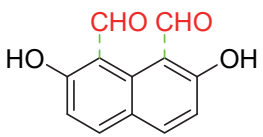

2i, $0 \%$
${ }^{a}$ Reaction conditions: naphthnols (2a) $(0.25 \mathrm{mmol})$, paraformaldehyde $(1.25$ mmol), ammonium acetate $(0.125 \mathrm{mmol})$ in acetic acid $(2.5 \mathrm{~mL})$ at $100{ }^{\circ} \mathrm{C}$ for 5 h. ${ }^{b}$ Isolated yield.

为探究反应的普遍性, 将䒺酚替换为苯酚和各种取 代的苯酚, 在标准条件下与多聚甲醛反应, 也得到了一 系列水杨醛类化合物, 结果见表 3 . 从表 3 可以看出, 苯 酚和酚羟基邻位不含取代基的大多数取代苯酚, 如 4-氯 苯酚、4-溴苯酚、4-甲基苯酚、4-甲氧基苯酚、3-硝基 苯酚和 2-氯苯酚, 都能与多聚甲醛反应得到相应的水杨 醛类化合物，产率在 $42 \% \sim 58 \%$ 之间 $(4 \mathrm{a} \sim \mathbf{4 g})$. 与萗酚 及其取代物相比, 苯酚的产物收率有明显降低. 这可能 是由于苯环上氧原子共轭效应没有䒺环上的强所导致 的. 有意思的是, 3-硝基苯酚生成的并不是空间位阻较
小的邻甲酰化产物 2-羟基-4-硝基苯甲醛，我们通过表 征数据分析并与文献[22]对比，确定甲酰化反应发生在 3-硝基与羟基之间的邻位上，即生成了 2-羟基-6-硝基苯 甲醛(4f, 52\%). 但是，当酚羟基的一侧邻位是氯原子时， 甲酰化反应不是发生在差基的另一侧，而是羟基的对位 (4g, 43\%). 这说明取代基的电子效应和空间位阻效应都 会影响反应的化学选择性. 与氨基䒺酚和芸二酚类似, 对氨基苯酚和对苯二酚在此反应中都不能得到相关产 物(4g 和 $4 \mathrm{~h}$ ), 可能是在该条件下, 这几个底物容易与甲 醛发生聚合反应.

表 3 苯酚底物的适用范围 $a, b$

Table 3 Scope of phenols

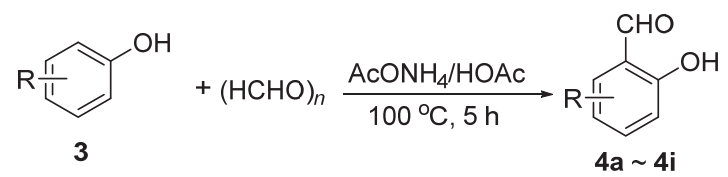

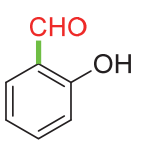

4a, $42 \%$<smiles>Cc1cc(C)c(O)c(O)c1</smiles><smiles>O=Cc1ccc(O)c(Cl)c1</smiles>

$4 \mathrm{~g}, 43 \%$<smiles>O=Cc1cc(Cl)ccc1O</smiles>

4b, $47 \%$<smiles>COc1ccc(O)c(C=O)c1</smiles>

$\mathrm{CHO}$
$\mathrm{H}_{2} \mathrm{~N}-$<smiles>Oc1ccccc1</smiles>

4h, $0 \%$<smiles>O=Cc1cc(Br)ccc1O</smiles>

4c, $56 \%$<smiles>O=Cc1c(O)cccc1[N+](=O)[O-]</smiles>

4f, $52 \%$ $\mathrm{CHO}$<smiles>O=Cc1cc(O)ccc1O</smiles>

4i, $0 \%$ ${ }^{a}$ Reaction conditions: phenols $(0.25 \mathrm{mmol})$, paraformaldehyde $(1.25 \mathrm{mmol})$,
ammonium acetate $(0.125 \mathrm{mmol})$ in acetic acid $(2.5 \mathrm{~mL})$ at $100{ }^{\circ} \mathrm{C}$ for $5 \mathrm{~h}$.
${ }^{b}$ Isolated yield.

\section{3 可能的反应机理}

在实验过程中，对该反应的机理进行了探索. 首先, 在氩气保护下, 将 2-萗酚(1a)、多聚甲醛、乙酸铵和冰 乙酸加入到圆底烧瓶中，于 $100{ }^{\circ} \mathrm{C}$ 下反应，薄层色谱 (TLC)跟踪反应完全后，发现得到的产物与空气中反应 得到的产物不一样。因此将该产物分离, 并进行 ${ }^{1} \mathrm{H}$ $\mathrm{NMR} 、{ }^{13} \mathrm{C} \mathrm{NMR}$ 表征，发现该化合物为 1-着圣基-2-萗 酚(5a), 收率为 $92 \%$. 将此产物在空气条件下, $100{ }^{\circ} \mathrm{C}$, 冰乙酸溶液中反应 $5 \mathrm{~h}$, 发现能生成目标产物 $\mathbf{2 a}$, 收率 为 $80 \%$ (Scheme 2, a). 还以 6-溴-2-荎酚 $(\mathbf{1 c})$ 作为底物, 分步进行了上述反应, 结果表明 6-溴-2-萗酚在氩气氛 中与多聚甲醛反应以 $82 \%$ 的产率生成了 1 -羟甲基-6-溴2-萗酚 $(5 \mathrm{c})$, 后者在空气中被氧化成了 2-羟基-6-溴萗-1甲醛，收率为 75\% (Scheme 2, b). 中间体化合物 $\mathbf{5 c}$ 的结 
构经 ${ }^{1} \mathrm{H}$ NMR, ${ }^{13} \mathrm{C}$ NMR 和高分辨质谱表征. 此外, 用湿 润的石荵试纸在反应瓶口检测, 发现试纸变成了浅蓝 色，这说明反应过程中确实有氨气生成.

通过以上实验, 结合相关文献 ${ }^{[23]}$, 我们提出了酚类 化合物的邻位甲酰化反应的可能机理, 见 Scheme 3 所 示. 首先, 多聚甲醛在加热条件下解聚, 形成甲醛分子. 甲醛与醋酸铵中的铵根离子反应脱除一分子水生成亚 胺正离子中间体 $\mathbf{I}$; 该正离子进攻 2-䒬酚(1a)的羟基邻 位形成 $\Pi$ 中间体, 中间体 $\Pi$ 释放一分子氨生成碳正离子 中间体 II; 氨继续与水中 $\mathrm{H}^{+}$结合形成 $\mathrm{NH}_{4}^{+}, \mathrm{NH}_{4}^{+}$再 与甲醛结合, 形成催化循环. 另一方面, $\mathrm{H}_{2} \mathrm{O}$ 作为亲核 试剂进攻中间体 II 得到烷基氧鎓离子 IV, IV 脱除质子 得到 1-羟甲基- $1 H$-䒬-2-酮(V). 接着, $\mathrm{H}_{2} \mathrm{O}$ 作为亲核试剂 再次进攻 $\mathbf{V}$ 的羰基碳原子, 得到偕二醇 VI. VI 发生消除 反应脱去一分子水, 得到 1-差圣甲基-2-菜酚(5a). 最后, 5a 在空气条件下氧化得到产物 2-羟基芸-1-甲醛(2a).

\section{2 结论}

以廉价的乙酸铵作为催化剂, 以固体多聚甲醛为甲 酰化试剂, 发展了一种高区域选择性、高效、经济合成 邻着基荎甲醛和邻羟基苯甲醛类化合物的方法. 该方法 无需添加任何金属催化剂，也不需要强酸和强碱性物 质，减少了对反应设备的腐蚀. 该方法反应体系简单， 条件温和, 反应速度快, 产率高, 后处理简单, 是一种 环境友好的合成邻羟基芳甲醛类化合物的方法.

\section{3 实验部分}

\section{1 仪器与试剂}

核磁共振谱用 BRUKER 公司 Mercury-Plus 400 $\mathrm{MHz}$ 和 $100 \mathrm{MHz}$ 的核磁共振仪，以四甲基硅烷(TMS)内 标, 忥代氯仿 $\left(\mathrm{CDCl}_{3}\right)$ 为溶剂; 采用北京泰克仪器有限 公司生产的 XT-5 型显微熔点测定仪测试化合物熔点.

(a)

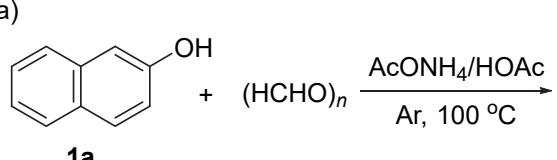<smiles></smiles>

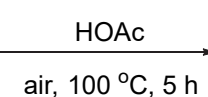

air, $100{ }^{\circ} \mathrm{C}, 5 \mathrm{~h}$<smiles>O=Cc1c(O)ccc2ccccc12</smiles>

2a

$80 \%$

(b)<smiles>CC(=O)ONC(=O)[C@H](O)OC=[Co+]</smiles><smiles>OCc1c(O)ccc2cc(Br)ccc12</smiles>
air, $100{ }^{\circ} \mathrm{C}, 5 \mathrm{~h} \mathrm{Br}$<smiles>O=Cc1c(O)ccc2cc(I)ccc12</smiles>

图式 2 控制实验

Scheme 2 Control experiments

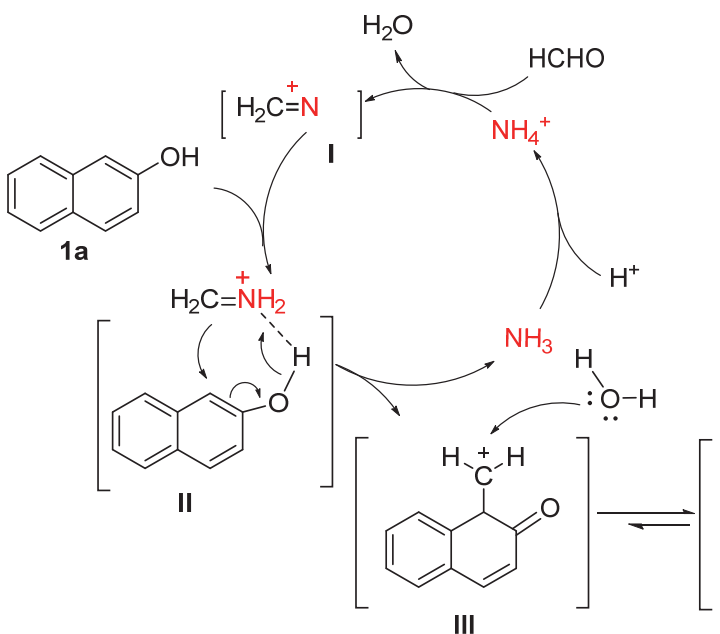

III<smiles>O=Cc1c(O)ccc2ccccc12</smiles><smiles>OCc1c(O)ccc2ccccc12</smiles>

图式 3 乙酸铵促进萗酚与多聚甲醛合成邻羟基萗甲醛的反应机理

Scheme 3 Possible reaction mechanism of ammonium acetate-catalyzed synthesis of ortho-hydroxynaphthalene formaldehydes from naphthols and paraformaldehyde 
高效薄层层析板购自烟台江友硅胶开发有限公司; 柱层析硅胶 $(300 \sim 400$ 目)购自青岛海洋化工厂; 石油 醚、乙酸乙酯均为工业品, 购自天津市福晨化学试剂厂; 其他试剂均为市售分析纯，使用前无需处理.

\section{2 化合物 $\mathbf{2 a} \sim \mathbf{2 g}, \mathbf{4 a} \sim \mathbf{4 g}, \mathbf{5 a}, \mathbf{5 c}$ 的合成}

以 2-羟基䒺-1-甲醛 $(\mathbf{2 a})$ 为例, 将 $0.25 \mathrm{mmol} 2$-菜酚 (1a)、 $1.25 \mathrm{mmol}$ 多聚甲醛、 $0.125 \mathrm{mmol}$ 乙酸铵、 $2.5 \mathrm{~mL}$ 冰乙酸置于 $50 \mathrm{~mL}$ 圆底烧瓶中, 于 $100{ }^{\circ} \mathrm{C}$ 下恒温摚拌, 并用薄层色谱法(TLC)跟踪反应. 反应完成后, 用乙酸 乙酯和水萃取有机相, 并用无水硫酸钠干燥. 最后, 以 石油醚/乙酸乙酯 $(V: V=20: 1)$ 作为流动相, 硅胶作为 固定相将有机相分离纯化得到目标产物 2-羟基萘-1-甲 醛. 采取相似的方法合成其它产物.

羟基萗-1-甲醛(2a): 黄色晶体, $36.6 \mathrm{mg}, 85 \%$. m.p. $76 \sim 80{ }^{\circ} \mathrm{C} \quad\left(\right.$ lit. $\left.^{[24]} 79 \sim 80{ }^{\circ} \mathrm{C}\right)$; ${ }^{1} \mathrm{H}$ NMR $(400 \mathrm{MHz}$, $\left.\mathrm{CDCl}_{3}\right) \delta: 10.08(\mathrm{~s}, 1 \mathrm{H}), 8.35(\mathrm{~d}, J=8.5 \mathrm{~Hz}, 1 \mathrm{H}), 7.98$ (d, $J=9.1 \mathrm{~Hz}, 1 \mathrm{H}), 7.80(\mathrm{~d}, J=8.1 \mathrm{~Hz}, 1 \mathrm{H}), 7.44$ (dd, $J=8.0$, $7.1 \mathrm{~Hz}, 1 \mathrm{H}), 7.62$ (t, $J=7.7 \mathrm{~Hz}, 1 \mathrm{H}), 7.26(\mathrm{~d}, J=0.6 \mathrm{~Hz}$, $1 \mathrm{H}), 7.14(\mathrm{~d}, J=9.1 \mathrm{~Hz}, 1 \mathrm{H}) ;{ }^{13} \mathrm{C}$ NMR $(100 \mathrm{MHz}$, $\left.\mathrm{CDCl}_{3}\right) \delta: 193.1,164.8,139.0,132.7,129.3,129.0,127.7$, 124.4, 119.0, 118.5, 111.1; IR (KBr) v: 3453, 2921, 1736 $\mathrm{cm}^{-1}$.

羟基菜-2-甲醛(2b): 黄色晶体, $37.0 \mathrm{mg}, 86 \%$. m.p. $50 \sim 53{ }^{\circ} \mathrm{C} \quad\left(\right.$ lit. $\left.^{[25]} 56 \sim 57{ }^{\circ} \mathrm{C}\right) ;{ }^{1} \mathrm{H}$ NMR $(400 \mathrm{MHz}$, $\left.\mathrm{CDCl}_{3}\right) \delta: 9.97(\mathrm{~s}, 1 \mathrm{H}), 8.51(\mathrm{~d}, J=8.1 \mathrm{~Hz}, 1 \mathrm{H}), 7.97$ (d, $J=8.2 \mathrm{~Hz}, 1 \mathrm{H}), 7.76(\mathrm{t}, J=7.5 \mathrm{~Hz}, 1 \mathrm{H}), 7.66 \sim 7.42(\mathrm{~m}$, 2H), $7.26(\mathrm{~s}, 1 \mathrm{H}), 5.48(\mathrm{~s}, 1 \mathrm{H}) ;{ }^{13} \mathrm{C}$ NMR $(100 \mathrm{MHz}$, $\left.\mathrm{CDCl}_{3}\right) \delta: 196.0,162.4,135.7,133.4,131.2,128.4,126.8$, 126.2, 124.9, 123.6, 123.1; IR (KBr) v: 3451, 2920, 1734 $\mathrm{cm}^{-1}$.

溴-2-羟基䒺-1-甲醛(2c): 黄色晶体, $48.9 \mathrm{mg}, 78 \%$. m.p. 149 $152{ }^{\circ} \mathrm{C}$ (lit. $\left.{ }^{[26]} 150{ }^{\circ} \mathrm{C}\right) ;{ }^{1} \mathrm{H}$ NMR (400 MHz, $\left.\mathrm{CDCl}_{3}\right) \delta: 10.62(\mathrm{~s}, 1 \mathrm{H}), 8.07(\mathrm{~d}, J=8.7 \mathrm{~Hz}, 1 \mathrm{H}), 7.90 \sim$ $7.63(\mathrm{~m}, 2 \mathrm{H}), 7.55(\mathrm{~d}, J=8.8 \mathrm{~Hz}, 1 \mathrm{H}), 7.38 \sim 7.06(\mathrm{~m}$, $2 \mathrm{H}) ;{ }^{13} \mathrm{C}$ NMR (100 MHz, $\left.\mathrm{CDCl}_{3}\right) \delta: 193.2,164.8,139.0$, $132.8,129.3,129.0,127.7,124.4,119.0,118.5,111.1$; IR (KBr) $v: 3493,3097,1711,875,667 \mathrm{~cm}^{-1}$.

4-氯-2-羟基苶-1-甲醛(2d): 黄色晶体，39.2 mg, 76\%. m.p. $1117 \sim 118{ }^{\circ} \mathrm{C}$ (lit. ${ }^{[27]} 118 \sim 119{ }^{\circ} \mathrm{C}$ ); ${ }^{1} \mathrm{H}$ NMR $\left(400 \mathrm{MHz}, \mathrm{CDCl}_{3}\right) \delta: 9.77(\mathrm{~s}, 1 \mathrm{H}), 8.34(\mathrm{~d}, J=8.0 \mathrm{~Hz}$, $1 \mathrm{H}), 8.07$ (d, $J=8.1 \mathrm{~Hz}, 1 \mathrm{H}), 7.67$ (t, $J=6.9 \mathrm{~Hz}, 1 \mathrm{H}), 7.49$ $(\mathrm{dd}, J=24.9,17.5 \mathrm{~Hz}, 2 \mathrm{H}), 7.17(\mathrm{~s}, 1 \mathrm{H}) ;{ }^{13} \mathrm{C}$ NMR $(100$ $\left.\mathrm{MHz}, \mathrm{CDCl}_{3}\right) \delta: 195.1,160.6,134.3,131.5,126.8,125.6$, 125.5, 124.6, 124.4, 122.3, 114.0; IR (KBr) v: 3475, 3055, $1741,885,757 \mathrm{~cm}^{-1}$. 4-溴-3-羟基菜-2-甲醛(2e)：黄色晶体，38.9 mg, 62\%. m.p. $115 \sim 118{ }^{\circ} \mathrm{C}$ (lit. ${ }^{[28]} 114 \sim 115{ }^{\circ} \mathrm{C}$ ); ${ }^{1} \mathrm{H}$ NMR $\left(400 \mathrm{MHz}, \mathrm{CDCl}_{3}\right) \delta: 10.75(\mathrm{~s}, 1 \mathrm{H}), 8.30(\mathrm{~d}, J=8.30 \mathrm{~Hz}$, 1H), 7.92 (d, $J=8.9 \mathrm{~Hz}, 1 \mathrm{H}), 7.74$ (d, $J=7.82 \mathrm{~Hz}, 1 \mathrm{H})$, $7.54(\mathrm{~d}, J=7.9 \mathrm{~Hz}, 1 \mathrm{H}), 7.38 \sim 7.06(\mathrm{~m}, 2 \mathrm{H}) ;{ }^{13} \mathrm{C} \mathrm{NMR}$ $\left(100 \mathrm{MHz}, \mathrm{CDCl}_{3}\right) \delta: 193.2,164.8,139.0,132.8,129.3$, $129.0,127.7,124.4,119.0,118.5,111.1$; IR (KBr) v: 3470, 2920, 1637, 810, 800, $614 \mathrm{~cm}^{-1}$.

羟基-4-硝基萘-2-甲醛(2f): 黄色晶体， $42.3 \mathrm{mg}$, 78\%. m.p. 161 $164{ }^{\circ} \mathrm{C}$ (lit. $\left.{ }^{[29]} 165{ }^{\circ} \mathrm{C}\right) ;{ }^{1} \mathrm{H}$ NMR (400 $\left.\mathrm{MHz} \mathrm{CDCl}_{3}\right) \delta: 9.96(\mathrm{~d}, J=1.2 \mathrm{~Hz}, 1 \mathrm{H}), 8.73$ (d, $J=8.8$ $\mathrm{Hz}, 1 \mathrm{H}), 8.52$ (d, $J=8.8 \mathrm{~Hz}, 1 \mathrm{H}), 7.90 \sim 7.63(\mathrm{~m}, 2 \mathrm{H})$, $7.19(\mathrm{~d}, J=0.9 \mathrm{~Hz}, 1 \mathrm{H}), 4.23(\mathrm{~s}, 1 \mathrm{H}) ;{ }^{13} \mathrm{C}$ NMR $(100 \mathrm{MHz}$, $\left.\mathrm{CDCl}_{3}\right) \delta: 195.2,165.7,134.2,133.8,130.8,128.8,127.6$, $127.4,125.0,123.9,119.8$; IR (KBr) v: 3567, 3057, 1700, $1540,1182,888 \mathrm{~cm}^{-1}$.

1-羟基-4-甲氧基萗-2-甲醛(2g)：黄色晶体, $37.4 \mathrm{mg}$, 74\%. m.p. 98 99 ${ }^{\circ} \mathrm{C}$ (lit. $\left.{ }^{[30]} 99 \sim 100{ }^{\circ} \mathrm{C}\right) ;{ }^{1} \mathrm{H}$ NMR (400 $\left.\mathrm{MHz}, \mathrm{CDCl}_{3}\right) \delta: 9.86(\mathrm{~s}, 1 \mathrm{H}), 8.36(\mathrm{~d}, J=8.3 \mathrm{~Hz}, 1 \mathrm{H})$ $8.15(\mathrm{~d}, J=8.3 \mathrm{~Hz}, 1 \mathrm{H}), 7.64 \sim 7.52(\mathrm{~m}, 2 \mathrm{H}), 7.19(\mathrm{~s}$, $1 \mathrm{H}), 6.67(\mathrm{~s}, 1 \mathrm{H}) ;{ }^{13} \mathrm{C} \mathrm{NMR}\left(100 \mathrm{MHz}, \mathrm{CDCl}_{3}\right) \delta: 195.8$, $156.6,148.5,130.8,130.2,130.2,128.8,126.7,124.2$, 122.0, 101.8, 55.7; IR (KBr) v: 3567, 3170, 2952, 2852, $1394,1129,842 \mathrm{~cm}^{-1}$.

2-羟基苯甲醛(4a) ${ }^{[31]}$ : 无色液体， $12.8 \mathrm{mg}, 42 \%$. ${ }^{1} \mathrm{H}$ NMR (400 MHz, $\left.\mathrm{CDCl}_{3}\right) \delta: 11.02$ (s, 1H), 9.89 (s, 1H), $7.56 \sim 7.50(\mathrm{~m}, 2 \mathrm{H}), 7.03 \sim 6.97(\mathrm{~m}, 2 \mathrm{H}) ;{ }^{13} \mathrm{C}$ NMR $(100$ $\left.\mathrm{MHz}, \mathrm{CDCl}_{3}\right) \delta: 196.6,161.6,136.9,133.7,120.6,119.8$, 117.5; IR (KBr) v: 3400, 2924, 2724, 1685, 1600, 1495 $\mathrm{cm}^{-1}$.

5-氯-2-羟基苯甲醛(4b): 淡黄色固体，18.4 mg, 47\%. m.p. 89 91 ${ }^{\circ} \mathrm{C}$ (lit. $\left.{ }^{[32]} 90 \sim 91{ }^{\circ} \mathrm{C}\right) ;{ }^{1} \mathrm{H}$ NMR $(400$ $\left.\mathrm{MHz} \mathrm{CDCl}_{3}\right) \delta: 10.92(\mathrm{~s}, 1 \mathrm{H}), 9.84(\mathrm{~s}, 1 \mathrm{H}), 7.53(\mathrm{t}, J=2.2$ $\mathrm{Hz}, 1 \mathrm{H}), 7.47$ (dt, $J=9.0,2.2 \mathrm{~Hz}, 1 \mathrm{H}), 6.95$ (dd, $J=8.7$ $\mathrm{Hz}, 1 \mathrm{H}) ;{ }^{13} \mathrm{C} \mathrm{NMR}\left(100 \mathrm{MHz}, \mathrm{CDCl}_{3}\right) \delta: 195.6,160.2$, 132.6, 124.8, 121.2, 119.6; IR (KBr) v: 3207, 2927, 2873, $1679,1657,1567,1470,1376,1301,1274,1156 \mathrm{~cm}^{-1}$.

2-羟基-5-溴苯甲醛(4c): 淡黄色固体, $28.0 \mathrm{mg}, 56 \%$. m.p. $103 \sim 105{ }^{\circ} \mathrm{C}$ (lit. $\left.{ }^{[33]} 102 \sim 105{ }^{\circ} \mathrm{C}\right) ;{ }^{1} \mathrm{H}$ NMR $(400$ $\left.\mathrm{MHz}, \mathrm{CDCl}_{3}\right) \delta: 10.93(\mathrm{~s}, 1 \mathrm{H}), 9.83(\mathrm{~s}, 1 \mathrm{H}), 7.67$ (d, $J=$ $2.5 \mathrm{~Hz}, 1 \mathrm{H}), 7.59$ (dd, $J=8.9,2.5 \mathrm{~Hz}, 1 \mathrm{H}), 6.90$ (d, $J=8.8$ $\mathrm{Hz}, 1 \mathrm{H}) ;{ }^{13} \mathrm{C} \mathrm{NMR}\left(100 \mathrm{MHz}, \mathrm{CDCl}_{3}\right) \delta: 195.6,160.7$, 139.8, 135.8, 121.8, 119.9, 111.5; IR (KBr) v: 3221, 2927, 2878, 1671, 1653, 1566, 1466, 1374, 1303, 1273, 1155 $\mathrm{cm}^{-1}$. 
2-羟基-5-甲基苯甲醛(4d)：黄色固体，15.3 mg， $45 \%$. m.p. $55 \sim 57{ }^{\circ} \mathrm{C}$ (lit. $\left.{ }^{[34]} 56{ }^{\circ} \mathrm{C}\right) ;{ }^{1} \mathrm{H}$ NMR $(400 \mathrm{MHz}$, $\left.\mathrm{CDCl}_{3}\right) \delta: 10.84(\mathrm{~s}, 1 \mathrm{H}), 9.86(\mathrm{~s}, 1 \mathrm{H}), 7.35 \sim 7.33(\mathrm{~m}, 2 \mathrm{H})$, $6.90(\mathrm{~d}, \quad J=9.2 \mathrm{~Hz}, 1 \mathrm{H}), 2.34(\mathrm{~s}, 3 \mathrm{H}) ;{ }^{13} \mathrm{C}$ NMR $(100$ $\left.\mathrm{MHz}, \mathrm{CDCl}_{3}\right) \delta: 195.5,163.3,142.3,137.6,122.0,118.7$, 116.2, 18.3; IR (KBr) v: 3044, 2927, 2891, 1667, 1642, 1617, 1444, 1377, 1325, 1286, $1234 \mathrm{~cm}^{-1}$.

2-羟基-5-甲氧基苯甲醛(4e) ${ }^{[35]}$ : 黄色液体, 18.6 mg, 49\%. ${ }^{1} \mathrm{H}$ NMR (400 MHz, $\left.\mathrm{CDCl}_{3}\right) \delta: 10.65$ (s, 1H), $9.84(\mathrm{~s}, 1 \mathrm{H}), 7.15 \sim 7.11(\mathrm{~m}, 1 \mathrm{H}), 6.98(\mathrm{~d}, J=3.2 \mathrm{~Hz}, 1 \mathrm{H})$, $6.92(\mathrm{~d}, J=8.8 \mathrm{~Hz}, 1 \mathrm{H}), 3.80$ (s, $3 \mathrm{H}) ;{ }^{13} \mathrm{C}$ NMR $(150 \mathrm{MHz}$, $\left.\mathrm{CDCl}_{3}\right) \delta: 196.1,156.0,152.7,125.2,120.0,118.7,115.2$, 55.9; IR (KBr) v: 3450, 3010, 2740, 2640, $1710 \mathrm{~cm}^{-1}$.

羟基-6-硝基苯甲醛(4f): 黄色固体, $21.7 \mathrm{mg}, 52 \%$. m.p. 51 52 ${ }^{\circ} \mathrm{C}$ (lit. $\left.{ }^{[36]} 53 \sim 54{ }^{\circ} \mathrm{C}\right) ;{ }^{1} \mathrm{H}$ NMR $(400 \mathrm{MHz}$, $\left.\mathrm{CDCl}_{3}\right) \delta: 12.11(\mathrm{~s}, 1 \mathrm{H}), 10.33(\mathrm{~s}, 1 \mathrm{H}), 7.62(\mathrm{t}, J=8.2 \mathrm{~Hz}$, $1 \mathrm{H}), 7.55(\mathrm{dd}, \quad J=7.9,1.2 \mathrm{~Hz}, 1 \mathrm{H}), 7.29(\mathrm{dd}, J=8.5,1.1$ $\mathrm{Hz}, 1 \mathrm{H}) ;{ }^{13} \mathrm{C} \mathrm{NMR}\left(100 \mathrm{MHz}, \mathrm{CDCl}_{3}\right) \delta: 194.0,163.4$, 151.4, 136.0, 124.4, 116.2, 112.4; IR (KBr) $v: 3086,2963$, 2928, 2847, 1650, 1568, 1533, 1439, 1388, 1340, 1267, $1164 \mathrm{~cm}^{-1}$.

氯-4-羟基苯甲醛(4g): 白色固体, $16.8 \mathrm{mg}, 43 \%$. m.p. $133 \sim 135{ }^{\circ} \mathrm{C}$ (lit. $\left.{ }^{[37]} 132 \sim 134{ }^{\circ} \mathrm{C}\right) ;{ }^{1} \mathrm{H}$ NMR $(400$ $\left.\mathrm{MHz},\left(\mathrm{CD}_{3}\right)_{2} \mathrm{SO}\right) \delta: 9.83(\mathrm{~s}, 1 \mathrm{H}), 7.89(\mathrm{~d}, J=1.2 \mathrm{~Hz}, 1 \mathrm{H})$, $7.73(\mathrm{dd}, J=5.6,1.2 \mathrm{~Hz}, 1 \mathrm{H}), 7.15(\mathrm{~d}, J=5.6 \mathrm{~Hz}, 1 \mathrm{H})$, $6.23(\mathrm{~s}, 1 \mathrm{H}) ;{ }^{13} \mathrm{C}$ NMR $\left(100 \mathrm{MHz}, \mathrm{DMSO}-d_{6}\right) \delta: 190.7$, 159.2, 132.1, 130.4, 129.6, 121.1, 117.2; IR (KBr) v: 3207, 2927, 2873, 1679, 1657, 1567, 1470, 1376, 1301, 1274, $1156 \mathrm{~cm}^{-1}$.

羟甲基-2-萗酚 (5a): 棕色固体, $40.0 \mathrm{mg}, 92 \%$. m.p. $193 \sim 195{ }^{\circ} \mathrm{C}$ (lit. $\left.{ }^{[38]} 192 \sim 193{ }^{\circ} \mathrm{C}\right) ;{ }^{1} \mathrm{H}$ NMR $(400 \mathrm{MHz}$, DMSO- $\left.d_{6}\right) \delta: 10.22(\mathrm{~s}, 1 \mathrm{H}), 8.23(\mathrm{~d}, J=8.4 \mathrm{~Hz}, 1 \mathrm{H}), 7.69$ (d, $J=7.6 \mathrm{~Hz}, 1 \mathrm{H}), 7.64(\mathrm{~d}, J=8.8 \mathrm{~Hz}, 1 \mathrm{H}), 7.31$ (d, $J=$ $8.8 \mathrm{~Hz}, 1 \mathrm{H}), 7.22(\mathrm{t}, J=7.6 \mathrm{~Hz}, 1 \mathrm{H}), 7.15(\mathrm{t}, J=7.6 \mathrm{~Hz}$, $1 \mathrm{H}), 4.74(\mathrm{~s}, 1 \mathrm{H}), 3.55$ (s, 2H); ${ }^{13} \mathrm{C}$ NMR $(100 \mathrm{MHz}$, $\left.\mathrm{CDCl}_{3}\right) \delta: 156.9,139.0,133.6,133.2,132.7,130.6,129.0$, 127.3, 124.6, 123.3, 25.6.

6-溴-1-羟甲基-2-萗酚 (5c): 白色固体, $51.8 \mathrm{mg}$, 82\%. m.p. $242 \sim 244{ }^{\circ} \mathrm{C} ;{ }^{1} \mathrm{H}$ NMR (400 MHz, DMSO- $d_{6}$ ) $\delta: 10.43(\mathrm{~s}, 1 \mathrm{H}), 8.29(\mathrm{~s}, 1 \mathrm{H}), 8.07(\mathrm{~d}, J=8.8 \mathrm{~Hz}, 1 \mathrm{H}), 7.90$ $(\mathrm{d}, J=2.0 \mathrm{~Hz}, 1 \mathrm{H}), 7.59$ (d, $J=9.2 \mathrm{~Hz}, 1 \mathrm{H}), 7.28$ (s, $J=$ $8.8 \mathrm{~Hz}, 1 \mathrm{H}), 4.64(\mathrm{~s}, 1 \mathrm{H}), 3.45$ (s, 2H); ${ }^{13} \mathrm{C}$ NMR $(100$ $\left.\mathrm{MHz}, \mathrm{CDCl}_{3}\right) \delta: 153.5,132.9,130.1,129.8,129.2,128.6$, $126.5,119.9,119.1,115.7,54.1$; HRMS (ESI) calcd for $\mathrm{C}_{11} \mathrm{H}_{10} \mathrm{O}_{2} \mathrm{Br}[\mathrm{M}+\mathrm{H}]^{+}$254.9831, found 254.9830.
辅助材料(Supporting Information) 化合物 2, 4 和 5 的 ${ }^{1} \mathrm{H}$ NMR 和 ${ }^{13} \mathrm{CNMR}$ 图谱. 这些材料可以免费从本刊网 站(http://sioc-journal.cn/)上下载.

\section{References}

[1] Vojacek, S.; Beese, K.; Alhalabi, Z.; Swyter, S.; Bodtke, A.; Schulzke, C.; Jung, M.; Sippl, W.; Link, A. Arch. Pharm. Chem. Life Sci. 2017, 350, e1700097.

[2] Lu, C.; Hu, J. H.; Wang, Z. C.; Xie, S. H.; Pan, T. T.; Huang, L.; Li, X. S. Med. Chem. Commun. 2018, 9, 1862.

[3] Xiao, J. M.; Feng, L.; Zhou, L. S.; Gao, H. Z.; Zhang, Y. L.; Yang, K. W. Eur. J. Med. Chem. 2013, 59, 150.

[4] Dobashi, Y.; Ohkatsu, Y. Polym. Degrad. Stab. 2008, 93, 436.

[5] Deb, M. L.; Pegu, C. D.; Borpatra, P. J.; Baruah, P. K. RSC $A d v$. 2016, 6, 40552.

[6] Goswami, S.; Maity, S.; Das, A. K.; Maity, A. C. Tetrahedron Lett. 2013, 54, 6631.

[7] Su, H. Y.; Huang, W. W.; Yang, Z. Y.; Lin, H.; Lin, H. K. J. Inclusion Phenom. Macrocyclic Chem. 2012, 72, 221.

[8] Long, F. J. Fluoresc. 2017, 27, 1331.

[9] Qin, J. C.; Fan, L.; Li, T. R.; Yang, Z. Y. Synth. Met. 2015, 199, 179.

[10] Ugi, I. Adv. Synth. Catal. 1997, 339, 499.

[11] Terret, N. K.; Gardener, M.; Gordon, D. W.; Kobylecki, R. J.; Steele, J. Tetrahedron 1995, 51, 8135.

[12] (a) Duff, J. C. J. Chem. Soc. 1941, 547. (b) Duff, J. C. J. Chem. Soc. 1944, 276.

[13] Reimer, K.; Tiemann, F. Chem. Ber. 1876, 9, 824.

[14] Hofsløkken, N. U.; Skattebøl, L. Acta Chem. Scand. 1999, 53, 258.

[15] Ma, K. R.; Huang, Z. Q.; Tang, X. D.; Zhang, P. C.; Shi, G. L. Chem. World 1987, 2, 58 (in Chinese).

(马克荣, 黄治清, 唐宪达, 张鹏程, 石桂林, 化学世界, 1987, 2, 58.)

[16] Xia, S. P.; Lv, Y. M.; Cheng, X. Y. Sichuan Chem. Ind. 2004, 7, 10 (in Chinese).

(夏士朋, 吕云妹, 成新燕, 四川化工, 2004, 7, 10.)

[17] Zhao, S. F.; Chen, N. Y.; Mei, S. H.; Sun, J. G.; Hao, W. D. J. Wuhan Univ. Technol. 2006, 28,73 (in Chinese).

(赵胜芳, 陈年友, 梅水华, 孙国建, 郝卫东, 武汉理工大学学 报, 2006, 28, 73.)

[18] Yue, X. L.; Wang, Z. Q.; Li, C. R.; Yang, Z. Y. Tetrahedron Lett. 2017, 58, 4532.

[19] Balali, E.; Shameli, A.; Naeimi, H.; Ghanbari, M. M. Orient. J. Chem. 2013, 29, 1611.

[20] Naeimi, H.; Zakerzadeh, E. New J. Chem. 2018, 42, 4590.

[21] (a) Wu, H.; Wu, J. C.; Du, Z. Chin. J. Org. Chem. 2017, 37, 1127 (in Chinese).

(吴慧, 武俊成, 杜正银, 有机化学, 2017, 37, 1127.)

(b) Hu, B.; Zhang, Y. M.; Wang, T. Y.; Du, Z. J. Liaocheng Univ. (Nat. Sci.) 2020, 33, 67 (in Chinese).

(胡贝, 张源民, 王天昀, 杜正银, 聊城大学学报 (自然科学版), 2020, 33, 67.)

(c) Zhang, W.; Dong, T.; Wang, T.; Du, Z. J. Liaocheng Univ. (Nat. Sci.) 2019, 32, 35 (in Chinese).

(张文莹, 董涛生, 王天昀, 杜正银, 聊城大学学报(自然科学版), 2019, 32, 35.)

[22] (a) Stover, J. S.; Shi, J.; Jin, W.; Vogt, P. K.; Boger, D. W. J. Am. Chem. Soc. 2009, 131, 3342 .

(b) Aridoss, G.; Laali, K. K. J. Org. Chem. 2011, 76, 8088.

[23] Ou, W.; Huang, P. Sci. China: Chem. 2020, 63, 11.

[24] Yue, X.-L.; Wang, Z.-Q.; Li, C.-R.; Yang, Z.-Y. Tetrahedron Lett. 2017, 58, 4532.

[25] Zhao, Q. G.; Wei, Q. C.; Xuan, M. D. Org. Lett. 2010, 12, 2202.

[26] Vojacek, S.; Beese, K.; Alhalabi, Z.; Swyter, S.; Bodtke, A.; Schulzke, C.; Jung, M.; Sippl, W.; Link, A. Arch. Pharm. Chem. Life Sci. 2017, 350, e1700097. 
[27] Burton, H. J. Chem. Soc. 1945, 280

[28] Muhammad, A.; David, O. M.; Paris, E. G. J. Chem. Soc., Perkin Trans. 1 2002, 1470.

[29] Andrew, C.; Benniston; Jérôme, F. Tetrahedron Lett. 2008, 49, 4292.

[30] Musgrave, O. C.; Skoyles, D. J. Chem. Res. 2006, 2006, 456.

[31] Asthanaa, M.; Syiemliehb, I.; Kumarc, A.; Lalb, R. A. Inorg. Chim. Acta 2020, 502, 119286.

[32] Li, L. J.; Fu, B.; Qiao, Y.; Wang, C.; Huang, Y. Y.; Liu, C. C.; Tian, C.; Du, J. L. Inorg. Chim. Acta 2014, 419, 135.

[33] Zysman-Colman, E.; Arias, K.; Siegel, J. S. Can. J. Chem. 2009, 87,440 .
[34] Balali, E.; Shameli, A.; Naeimi, H.; Ghanbari, M. Orient. J. Chem. 2013, 29, 1611.

[35] Antúnez, D. J. B.; Greenhalgh, M. D.; Fallan, C.; Slawin, A. M. Z.; Smith, A. D. Org. Biomol. Chem. 2016, 14, 7268.

[36] Horton, D. A.; Bourne, G. T.; Coughlan, J.; Kaiser, S. M.; Jacobs, C. M.; Jones, A.; Ruhmann, A.; Turnerb, J. Y.; Smythe, M. L. Org. Biomol. Chem. 2008, 6, 1386.

[37] Knuutlnen, J. S.; Kolehmainen, E. T. J. Chem. Eng. Data 1983, 28, 139.

[38] Casiraghi, G.; Casnati, G.; Puglia, G.; Sartori, G. Synthesis 1980, 124.

(Zhao, C.) 